\title{
Impact of a XENONnT signal on LHC dijet searches
}

\author{
Sebastian Baum, ${ }^{a, b}$ Riccardo Catena $^{c}$ and Martin B. Krauss ${ }^{c, d}$ \\ ${ }^{a}$ Oskar Klein Centre, Department of Physics, Stockholm University, AlbaNova, \\ SE-10691 Stockholm, Sweden \\ ${ }^{b}$ Nordita, KTH Royal Institute of Technology and Stockholm University, \\ Roslagstullsbacken 23, SE-10691 Stockholm, Sweden \\ ${ }^{c}$ Department of Physics, Chalmers University of Technology, \\ SE-412 96 Göteborg, Sweden \\ ${ }^{d}$ Dipartimento di Matematica e Fisica, Università di Roma Tre, \\ Via della Vasca Navale 84, 00146 Rome, Italy \\ E-mail: sbaum@fysik.su.se, catena@chalmers.se, \\ martin.krauss@chalmers.se
}

ABSTRACT: It is well-known that dark matter (DM) direct detection experiments and the LHC are complementary, since they probe physical processes occurring at different energy scales. And yet, there are aspects of this complementarity which are still not fully understood, or exploited. For example, what is the impact that the discovery of DM at XENONnT would have on present and future searches for DM in LHC final states involving a pair of hadronic jets? In this work we investigate the impact of a XENONnT signal on the interpretation of current dijet searches at the LHC, and on the prospects for dijet signal discovery at the High-Luminosity (HL) LHC in the framework of simplified models. Specifically, we focus on a general class of simplified models where DM can have spin 0, $1 / 2$ or 1 , and interacts with quarks through the exchange of a scalar, pseudo-scalar, vector, or pseudo-vector mediator. We find that exclusion limits on the mediator's mass and its coupling to quarks from dijet searches at the LHC are significantly affected by a signal at XENONnT, and that $\mathcal{O}(100)$ signal events at XENONnT would drastically narrow the region in the parameter space of simplified models where a dijet signal can be discovered at $5 \sigma$ C.L. at the HL-LHC.

Keywords: Cosmology of Theories beyond the SM, Effective Field Theories, Beyond Standard Model

ARXIV EPRINT: 1812.01594 


\section{Contents}

1 Introduction 1

2 Theoretical framework 3

$\begin{array}{lll}3 & \text { Statistical methods } & 7\end{array}$

4 Analysis $\quad 8$

4.1 Impact of a XENONnT signal on LHC dijet exclusion limits 10

4.2 Impact of a XENONnT signal on LHC dijet $5 \sigma$ discovery contours $\quad 12$

5 Conclusion $\quad 15$

$\begin{array}{ll}\text { A Lagrangians for simplified dark matter models } & 17\end{array}$

$\begin{array}{lll}\text { A.1 Scalar dark matter } S & 17\end{array}$

$\begin{array}{lll}\text { A.2 } & \text { Fermionic dark matter } \chi & 18\end{array}$

$\begin{array}{ll}\text { A.3 Vector dark matter } X_{\mu} & 19\end{array}$

\section{Introduction}

Observations in a wide range of astronomical and cosmological systems show that the Universe contains about five times as much dark matter (DM) as baryonic matter [1]. While the nature of DM remains unknown, the hypothesis that DM is made of yet unidentified particles is the one explored most extensively [2]. If DM is made of Weakly Interacting Massive Particles (WIMPs), as predicted by many theories beyond the Standard Model (BSM) addressing the hierarchy problem, e.g. supersymmetric extension of the Standard Model (SM), it can potentially be observed in the next stage of direct detection and particle collider experiments [3]. DM direct detection experiments primarily search for nuclear recoils induced by the non-relativistic scattering of Milky Way DM particles in low-background detectors located deep underground $[4,5]$. The null results of present DM direct detection experiments place severe constraints on the strength with which DM couples to the fundamental constituents of matter. The most stringent limits from direct detection experiments on the strength of DM-nucleus interactions for WIMPs heavier than about $10 \mathrm{GeV}$ are currently set by the XENON1T collaboration [6], improving on previous results from LUX [7] and PandaX-II [8]. They reported a 90\% C.L. exclusion limit on the elastic spin-independent DM-nucleon scattering cross-section with a minimum of $4.1 \times 10^{-47} \mathrm{~cm}^{2}$ around a DM mass of $30 \mathrm{GeV}$. XENONnT, the upgrade of XENON1T, is expected to operate from 2019 onwards using about 7 ton of ultra-pure liquid xenon as target material [9]. Note that although we use XENONnT as an example for the purposes of this work, the LZ [10] and PandaX-4T [11] experiments plan to achieve similar sensitivity on comparable time scales as XENONnT. Pursuing a complementary approach to direct detection experiments, the Large Hadron Collider (LHC) at CERN with data analysed by the ATLAS 
and CMS collaborations is also searching for DM. At the LHC, DM can be produced in the collision of energetic protons, and its production be inferred through the observation of missing transverse momentum in the final state of such collisions. The next run of the LHC (Run 3) will start in 2021 [12]. It will operate at the centre-of-mass energy of $\sqrt{s}=13 \mathrm{TeV}$ and reach the expected integrated luminosity of $300 \mathrm{fb}^{-1}$ in 2023. The LHC Run 3 will be followed by the high luminosity run of the LHC (HL-LHC), which is expected to start in 2026, reaching an integrated luminosity of $3000 \mathrm{fb}^{-1}$.

LHC data on processes that might involve DM have been interpreted within different theoretical frameworks. Ultraviolet (UV) complete theories (with a focus on supersymmetric theories) and Effective Field Theories (EFTs) for DM-quark and -gluon interactions have been used extensively in the analyses of the LHC Run 1 results (see [13], and references therein). Limitations in the applicability of an EFT approach to the interpretation of LHC data led to a change of framework for the interpretation of results from the LHC Run 2 [14], which were primarily analysed within the framework of simplified models (see [15], and references therein). By construction, in simplified models for DM, the SM is extended by the DM particle and one single mediator particle only. The latter is responsible for the interactions of DM with SM particles, for example, quarks and gluons. When the momentum transferred in a proton-proton collision at the LHC is smaller than the mediator mass (which is not a priori true), the mediator can be "integrated out" and simplified models converge to EFTs. Compared to an EFT approach, the use of simplified models allows for a more complex analysis of the LHC data, especially in the study of processes which involve the mediator explicitly, as in the case of final states including hadronic jets produced by the decay of the mediator into a quark pair. While simplified models are generically not UV complete, and their applicability is subject to constraints from unitarity and anomaly cancellation, see e.g. [16, 17], they provide a good compromise between simplicity and completeness.

While processes that directly involve the DM particle are obviously important to reconstruct DM mass and coupling constants at the LHC and have therefore been studied, e.g., in monojet searches $[18,19]$, events involving the mediator particle alone can be used to obtain important information on the underlying DM model as well [20]. In this context, dijet searches play a special role [21]. Within the framework of simplified models for DMquark interactions, neutral mediators can be resonantly produced during a proton-proton collision and then decay into a pair of quarks. Due to hadronization these will be seen by a detector as a pair of hadronic jets. The analysis of dijet events at ATLAS and CMS has been one of the main channels in the search for new physics at the LHC. Recently, ATLAS has published results for generic (high-mass) dijet searches from $37 \mathrm{fb}^{-1}$ of data collected during 2015 and 2016 [22], whereas CMS has presented results for $36 \mathrm{fb}^{-1}$ of data from the 2016 dataset $[23,24]$, as well as preliminary results for $78 \mathrm{fb}^{-1}$ of data from the combined 2016 and 2017 datasets [25]. ${ }^{1}$ In these studies, both collaborations have presented results only for a subset of all possible simplified models, mainly focusing on a vector mediator and fermionic DM.

\footnotetext{
${ }^{1}$ ATLAS has published data including the 2017 data set only for a dijet search requiring an additional isolated lepton in the final state [26].
} 
Direct detection experiments and the LHC are complementary since they explore physical processes occurring at different energy scales, see e.g. ref. [27]. Nevertheless, there are aspects of this complementarity which are still not fully understood, or exploited. For example, what is the impact of a signal at XENONnT on present and future searches for DM and new physics in general at the LHC? In this work we investigate the impact of a XENONnT signal on the interpretation of current dijet searches at the LHC, and on the prospects for dijet signal discovery at the HL-LHC. As a theoretical framework, we use a general class of simplified models where DM can have spin $0,1 / 2$ or 1 , and interacts with quarks through the exchange of scalar, pseudo-scalar, vector, or pseudo-vector mediators. This study extends our previous work [28], where we focused on the impact of a XENONnT signal on monojet searches at the LHC. We find that exclusion limits on mediator parameters from dijet searches at the LHC are significantly affected by a signal at XENONnT, and that $\mathcal{O}(100)$ signal events at XENONnT dramatically narrow the region in the parameter space of simplified models where a dijet signal can be discovered at $5 \sigma$ C.L.

We will show that there is an interesting interplay at work between direct detection and dijet searches, since the mass of the mediator and its couplings to quarks and DM are correlated when a direct detection signal is observed. If one fixes the mediator mass and one coupling, the other coupling will be fully determined by the direct detection signal. Since direct detection is sensitive to the product of the couplings of the mediator to quarks and to DM only, it is possible that the dijet signal is too small to be detectable if the mediator-DM coupling is large and the mediator-quark coupling small. However, we will show that sizable regions of parameter space remain, where both a signal in direct detection and in dijet searches at the LHC can potentially be detected. Furthermore, the relation between direct detection and dijet signals is model dependent. Thus, the combination of the two approaches allows one to gain additional information about the model and to break degeneracies between different models which appear when considering either direct detection or dijet searches only.

The remaining part of this article is organised as follows. In sections 2 and 3 we review theoretical framework and statistical methods. Our analysis of the impact of a XENONnT signal on LHC dijet searches is illustrated in section 4 . We conclude in section 5 and list useful equations in appendix A.

\section{Theoretical framework}

The theoretical framework used in this work consists of a set of simplified models where DM can have spin $0,1 / 2$ or 1 , and interacts with quarks through the exchange of scalar, pseudo-scalar, vector or pseudo-vector mediators [29]. Beyond the spin of the DM candidate and the mediator, the simplified models are specified by the Lorentz structure of the corresponding interaction vertices, and four free parameters. For each simplified model, the four free parameters are the DM particle and mediator masses, $m_{\mathrm{DM}}$ and $m_{\text {med }}$, respectively, and two coupling constants: one for the DM-DM-mediator vertex, $g_{\mathrm{DM}}$, and the 
second one for the quark-quark-mediator vertex, $g_{q} \cdot{ }^{2}$ Presenting our results, we specifically focus on nine simplified models characterised by the interaction Lagrangians

$$
\begin{aligned}
& \mathcal{L}_{1}=-h_{1} \bar{q} q \phi-g_{1} m_{S} S^{\dagger} S \phi ; \quad(S \otimes S)_{0} \\
& \mathcal{L}_{2}=-h_{3}\left(\bar{q} \gamma_{\mu} q\right) G^{\mu}-i g_{4}\left(S^{\dagger} \partial_{\mu} S-\partial_{\mu} S^{\dagger} S\right) G^{\mu} ; \quad(V \otimes i \partial)_{0} \\
& \mathcal{L}_{3}=-h_{1} \phi \bar{q} q-\lambda_{1} \phi \bar{\chi} \chi ; \quad(S \otimes S)_{1 / 2} \\
& \mathcal{L}_{4}=-h_{1} \phi \bar{q} q-i \lambda_{2} \phi \bar{\chi} \gamma^{5} \chi ; \quad(S \otimes P S)_{1 / 2} \\
& \mathcal{L}_{5}=-h_{3} \bar{q} \gamma_{\mu} q G^{\mu}-\lambda_{3} \bar{\chi} \gamma^{\mu} \chi G_{\mu} ; \quad(V \otimes V)_{1 / 2} \\
& \mathcal{L}_{6}=-h_{3} \bar{q} \gamma_{\mu} q G^{\mu}-\lambda_{4} \bar{\chi} \gamma^{\mu} \gamma^{5} \chi G_{\mu} ; \quad(V \otimes A)_{1 / 2} \\
& \mathcal{L}_{7}=-h_{4} \bar{q} \gamma_{\mu} \gamma^{5} q G^{\mu}-\lambda_{4} \bar{\chi} \gamma^{\mu} \gamma^{5} \chi G_{\mu} ; \quad(A \otimes A)_{1 / 2} \\
& \mathcal{L}_{8}=-h_{1} \phi \bar{q} q-b_{1} m_{X} \phi X_{\mu}^{\dagger} X^{\mu} ; \quad(S \otimes S)_{1} \\
& \mathcal{L}_{9}=-h_{3} G_{\mu} \bar{q} \gamma^{\mu} q-i b_{5}\left(X_{\nu}^{\dagger} \partial_{\mu} X^{\nu}-X^{\nu} \partial_{\mu} X_{\nu}^{\dagger}\right) G^{\mu} ; \quad(V \otimes i \partial)_{1}
\end{aligned}
$$

where $g_{q}=h_{1}, h_{2}, h_{3}$ or $h_{4}$ and $g_{\mathrm{DM}}=\lambda_{1}, \lambda_{2}, \lambda_{3}, \lambda_{4}, g_{1}, g_{4}, b_{1}$ or $b_{5}$, depending on the model. In eq. (2.1), scalar, fermionic and vector DM are described by the complex scalar field $S$, the spinor field $\chi$ and the complex vector field $X_{\nu}$, respectively. Quarks spinors are denoted by $q$, and a summation on quark flavours is understood. Next to each interaction Lagrangian, we have introduced a "label" which will be used in the following to refer to the corresponding simplified model. For example, $(S \otimes S)_{0}$, is the label for spin 0 DM coupling to quarks via a scalar DM-DM-mediator vertex and a scalar quark-quarkmediator vertex. Similarly, $(V \otimes i \partial)_{1}$ refers to spin $1 \mathrm{DM}$ coupling to quarks via a vector quark-quark-mediator vertex and a derivative DM-DM-mediator vertex. In the remaining cases, the letter $A$ refers to axial coupling and $P S$ to pseudo-scalar coupling. As we will explain later, the simplified models in eq. (2.1) form the subset of models from [29] that are compatible with the discovery of $\mathcal{O}(100)$ signal events at XENONnT and the current search for narrow resonances in dijet final states at the LHC. For completeness, the full set of simplified models from [29] is reported in appendix A. These models were introduced in the context of DM direct detection [29], and later applied to LHC monojet analyses [28] and DM relic density calculations [30].

For each simplified model in eq. (2.1), we simulate dijet signals at the LHC, and calculate the corresponding dijet invariant mass spectrum, by using the chain of numerical programs

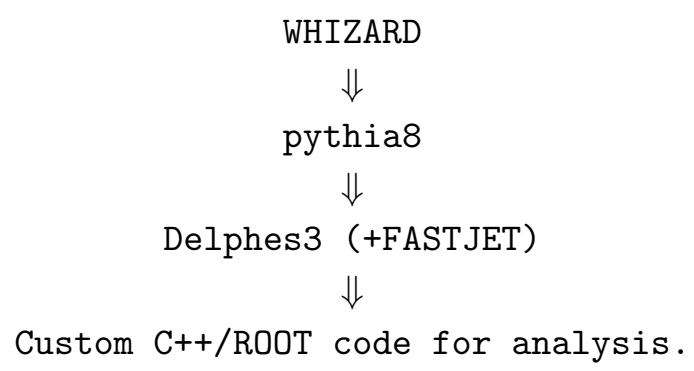

\footnotetext{
${ }^{2}$ Note that in particular all relevant quantities for dijet searches, i.e. the mediator's production cross section, its width, and its branching ratios, are fixed by these four parameters.
} 
We use WHIZARD $[31,32]$ with model files implementing the simplified models to generate the hard processes

$$
p, p \rightarrow \text { Mediator } \rightarrow \bar{q} q+X
$$

where $X$ stands for additional SM particles and $\bar{q} q$ can be any pair of quarks with the same flavor. We use parton distribution functions from the CT14lo set as obtained from LHAPDF6 [33], pythia8 [34] is used for showering and hadronization, Delphes3 [35] for (CMS) detector simulation, and FAST JET [36] for jet reconstruction. We use our own C++ code and ROOT [37] libraries to analyse the signal. We discard events where one (or both) of the leading jets deposit more than $90 \%$ of their respective total calorimetric energy in the electromagnetic calorimeter. See [38] for a more detailed discussion of our collider simulations.

For each simplified model in eq. (2.1), we are also interested in the rate of DM-nucleus scattering events at XENONnT. The expected rate per unit detector mass can be written as

$$
\frac{\mathrm{d} R}{\mathrm{~d} E_{R}}=\sum_{T} \xi_{T} \frac{\rho_{\chi}}{m_{\chi} m_{T}} \int_{|\mathbf{v}| \geq v_{\min }} \mathrm{d}^{3} v|\mathbf{v}| f(\mathbf{v}) \frac{\mathrm{d} \sigma_{T}}{\mathrm{~d} E_{R}}\left(|\mathbf{v}|^{2}, E_{R}\right),
$$

where $v_{\min }$ is the minimum DM speed to deposit an energy $E_{R}$ in the detector, $\mathrm{d} \sigma_{T} / \mathrm{d} E_{R}$ is the differential cross section for DM-nucleus scattering, $\rho_{\chi}$ is the local DM density, and $f(\mathbf{v})$ is the local DM velocity distribution in the detector rest frame. In the sum in eq. (2.3), we consider the seven most abundant xenon isotopes. Their mass fraction is denoted here by $\xi_{T}$. In order to calculate the expected rate of DM-nucleus scattering events at XENONnT for the simplified models in eq. (2.1), we proceed as follows. First, we analytically calculate the amplitude for DM scattering on free nucleons as described in detail in [28, 40-45]. From these amplitudes, we extract the coupling constants for DM-nucleon interactions, which are related to the ones in the Lagrangians in eq. (2.1), as illustrated in table 1. We then use the coupling constants in table 1 as an input for the package DMFormFactor [46], which provides a generalized set of nuclear response functions that properly treat velocity dependent WIMP interactions, and from which we extract the rate of DM-nucleus scattering events at XENONnT as an output. The result of this calculation depends on the local DM density and velocity distribution. For the DM velocity distribution in the detector rest frame, we assume a Maxwellian velocity distribution with a circular speed of $220 \mathrm{~km} \mathrm{~s}^{-1}$ for the local standard of rest, and a galactic escape velocity of $544 \mathrm{~km} \mathrm{~s}^{-1}$. Finally, for the local DM density we adopt the value $0.4 \mathrm{GeV} \mathrm{cm}^{-3}$.

The coupling constants in table 1 are the coefficients of quantum mechanical operators defining the non-relativistic effective theory of DM-nucleon interactions [39, 47, 48]. In this framework, DM-nucleon interaction operators are denoted by $\hat{\mathcal{O}}_{i}^{(N)}$, or just $\hat{\mathcal{O}}_{i}$ for simplicity. We list them in table 2 for completeness. They are expressed in terms of the basic invariants under Galilean transformations and Hermitian conjugation, namely: the momentum transfer operator, $\hat{\boldsymbol{q}}$, the transverse relative velocity operator $\hat{\boldsymbol{v}}^{\perp}$, the nucleon and DM spin operators, $\hat{\boldsymbol{S}}_{N}$ and $\hat{\boldsymbol{S}}_{\chi}$, respectively, and the identities in the nucleon and DM spin spaces, $\mathbb{1}_{\chi}$ and $\mathbb{1}_{N}$. In table $2, m_{N}$ is the nucleon mass, and all interaction operators have the same mass dimension. Within this notation, standard spin-independent and spindependent interactions correspond to the operators $\hat{\mathcal{O}}_{1}$ and $\hat{\mathcal{O}}_{4}$, respectively. The operators 


\begin{tabular}{|ll|}
\hline Spin $0 \mathrm{DM}$ & Non-relativistic coefficients different from zero \\
$(S \otimes S)_{0}$ & $c_{1}^{(N)}=\frac{h_{1}^{N} g_{1}}{M_{\Phi}^{2}}$ \\
$(V \otimes i \partial)_{0}$ & $c_{1}^{(N)}=-2 \frac{h_{3}^{N} g_{4}}{M_{G}^{2}}$ \\
\hline spin $1 / 2 \mathrm{DM}$ & Non-relativistic coefficients different from zero \\
$(S \otimes S)_{1 / 2}$ & $c_{1}^{(N)}=\frac{h_{1}^{N} \lambda_{1}}{M_{\Phi}^{2}}$ \\
$(S \otimes P S)_{1 / 2}$ & $c_{11}^{(N)}=-\frac{h_{1}^{N} \lambda_{2}}{M_{\Phi}^{2}} \frac{m_{N}}{m_{\chi}}$ \\
$(V \otimes V)_{1 / 2}$ & $c_{1}^{(N)}=-\frac{h_{3}^{N} \lambda_{3}}{M_{G}^{2}}$ \\
$(V \otimes A)_{1 / 2}$ & $c_{8}^{(N)}=-2 \frac{h_{3}^{N} \lambda_{4}}{M_{G}^{2}} ; \quad c_{9}^{(N)}=-2 \frac{h_{3}^{N} \lambda_{4}}{M_{G}^{2}}$ \\
$(A \otimes A)_{1 / 2}$ & $c_{4}^{(N)}=4 \frac{h_{4}^{N} \lambda_{4}}{M_{G}^{2}}$ \\
\hline spin $1 \mathrm{DM}$ & Non-relativistic coefficients different from zero \\
$(S \otimes S)_{1}$ & $c_{1}^{(N)}=\frac{b_{1} h_{1}^{N}}{M_{\Phi}^{2}}$ \\
$(V \otimes i \partial)_{1}$ & $c_{1}^{(N)}=-2 \frac{h_{3}^{N} b_{5}}{M_{G}^{2}}$ \\
\hline
\end{tabular}

Table 1. Relation between the coupling constants of non-relativistic operators from table 2 (in the proton/neutron basis) and simplified models in this study (see eq. (2.1) and appendix A for their Lagrangians). In the case of spin $1 \mathrm{DM}$, we do not consider non-relativistic operators that depend on the symmetric combination of polarisation vectors denoted by $\mathcal{S}$ in table 2 .

$\hat{\mathcal{O}}_{2}$ and $\hat{\mathcal{O}}_{16}$ do not appear in table 2 for the following reasons: the former is quadratic in $\hat{\boldsymbol{v}}^{\perp}$ (while the effective theory expansion in [39] is truncated at second order in $\hat{\boldsymbol{q}}$ and at linear order in $\hat{\boldsymbol{v}}^{\perp}$ ) and the latter is not independent, being a linear combination of the interaction operators $\hat{\mathcal{O}}_{12}$ and $\hat{\mathcal{O}}_{15}$. Finally, the operators $\hat{\mathcal{O}}_{17}$ and $\hat{\mathcal{O}}_{18}$ in table 2 can only arise for spin $1 \mathrm{DM}$, and $\mathcal{S}$ is a symmetric combination of spin 1 polarisation vectors [29]. In terms of the interaction operators $\hat{\mathcal{O}}_{i}$ in table 2, each simplified model in eq. (2.1) generates an Hamiltonian for non-relativistic DM-nucleon interactions, $\mathscr{H}$, which can be expressed as follows

$$
\mathscr{H}=\sum_{N=p, n} \sum_{i} c_{i}^{(N)} \hat{\mathcal{O}}_{i}^{(N)}
$$

where $c_{i}^{(p)}$ and $c_{i}^{(n)}$ are the coupling constants for protons and neutrons, respectively. For example, the simplified model characterised by fermionic DM and a vector mediator of mass $M_{G}$ that couples to DM with coupling constant $g_{\mathrm{DM}}=\lambda_{3}$ and to quarks with (a universal) coupling constant $g_{q}=h_{3}$ generates the operator $\hat{\mathcal{O}}_{1}$ in the non-relativistic limit. In this case, $c_{1}^{(N)}=-h_{3}^{N} \lambda_{3} / M_{G}^{2}$, where the nucleon-level and quark-level coupling constants, $h_{3}^{N}$ and $h_{3}$, are related by $h_{3}^{N}=3 h_{3}$. For expressions relating $h_{i}^{(N)}$ to $h_{i}$, with $i=1,2,4$, we refer to [29]. In the non-relativistic limit, some of the simplified models in eq. (2.1) generate a linear combination of operators in table 2 (see table 1). However, for 


\begin{tabular}{|ll}
\hline$\hat{\mathcal{O}}_{1}=\mathbb{1}_{\chi} \mathbb{1}_{N}$ & $\hat{\mathcal{O}}_{10}=i \hat{\mathbf{S}}_{N} \cdot \frac{\hat{\mathbf{q}}}{m_{N}} \mathbb{1}_{\chi}$ \\
$\hat{\mathcal{O}}_{3}=i \hat{\mathbf{S}}_{N} \cdot\left(\frac{\hat{\mathbf{q}}}{m_{N}} \times \hat{\mathbf{v}}^{\perp}\right) \mathbb{1}_{\chi}$ & $\hat{\mathcal{O}}_{11}=i \hat{\mathbf{S}}_{\chi} \cdot \frac{\hat{\mathbf{q}}}{m_{N}} \mathbb{1}_{N}$ \\
$\hat{\mathcal{O}}_{4}=\hat{\mathbf{S}}_{\chi} \cdot \hat{\mathbf{S}}_{N}$ & $\hat{\mathcal{O}}_{12}=\hat{\mathbf{S}}_{\chi} \cdot\left(\hat{\mathbf{S}}_{N} \times \hat{\mathbf{v}}^{\perp}\right)$ \\
$\hat{\mathcal{O}}_{5}=i \hat{\mathbf{S}}_{\chi} \cdot\left(\frac{\hat{\mathbf{q}}}{m_{N}} \times \hat{\mathbf{v}}^{\perp}\right) \mathbb{1}_{N}$ & $\hat{\mathcal{O}}_{13}=i\left(\hat{\mathbf{S}}_{\chi} \cdot \hat{\mathbf{v}}^{\perp}\right)\left(\hat{\mathbf{S}}_{N} \cdot \frac{\hat{\mathbf{q}}}{m_{N}}\right)$ \\
$\hat{\mathcal{O}}_{6}=\left(\hat{\mathbf{S}}_{\chi} \cdot \frac{\hat{\mathbf{q}}}{m_{N}}\right)\left(\hat{\mathbf{S}}_{N} \cdot \frac{\hat{\mathbf{q}}}{m_{N}}\right)$ & $\hat{\mathcal{O}}_{14}=i\left(\hat{\mathbf{S}}_{\chi} \cdot \frac{\hat{\mathbf{q}}}{m_{N}}\right)\left(\hat{\mathbf{S}}_{N} \cdot \hat{\mathbf{v}}^{\perp}\right)$ \\
$\hat{\mathcal{O}}_{7}=\hat{\mathbf{S}}_{N} \cdot \hat{\mathbf{v}}^{\perp} \mathbb{1}_{\chi}$ & $\hat{\mathcal{O}}_{15}=-\left(\hat{\mathbf{S}}_{\chi} \cdot \frac{\hat{\mathbf{q}}}{m_{N}}\right)\left[\left(\hat{\mathbf{S}}_{N} \times \hat{\mathbf{v}}^{\perp}\right) \cdot \frac{\hat{\mathbf{q}}}{m_{N}}\right]$ \\
$\hat{\mathcal{O}}_{8}=\hat{\mathbf{S}}_{\chi} \cdot \hat{\mathbf{v}}^{\perp} \mathbb{1}_{N}$ & $\hat{\mathcal{O}}_{17}=i \frac{\hat{\mathbf{q}}}{m_{N}} \cdot \mathcal{S} \cdot \hat{\mathbf{v}}^{\perp} \mathbb{1}_{N}$ \\
$\hat{\mathcal{O}}_{9}=i \hat{\mathbf{S}}_{\chi} \cdot\left(\hat{\mathbf{S}}_{N} \times \frac{\hat{\mathbf{q}}}{m_{N}}\right)$ & $\hat{\mathcal{O}}_{18}=i \frac{\hat{\mathbf{q}}}{m_{N}} \cdot \mathcal{S} \cdot \hat{\mathbf{S}}_{N}$ \\
\hline
\end{tabular}

Table 2. Quantum mechanical operators defining the non-relativistic effective theory of DMnucleon interactions [39]. The operators are expressed in terms of the basic invariants under Galilean transformations: the momentum transfer, $\hat{\boldsymbol{q}}$, the transverse relative velocity operator $\hat{\boldsymbol{v}}^{\perp}$, the nucleon and DM spin operators, denoted by $\hat{\boldsymbol{S}}_{N}$ and $\hat{\boldsymbol{S}}_{\chi}$, respectively, and the identities in the nucleon and DM spin spaces, $\mathbb{1}_{\chi}$ and $\mathbb{1}_{N}$. All operators have the same mass dimension, and $m_{N}$ is the nucleon mass. Standard spin-independent and spin-dependent interactions correspond to the operators $\hat{\mathcal{O}}_{1}$ and $\hat{\mathcal{O}}_{4}$, respectively, while $\mathcal{S}$ is a symmetric combination of spin 1 polarisation vectors [29]. The operators $\hat{\mathcal{O}}_{17}$ and $\hat{\mathcal{O}}_{18}$ can only arise for spin 1 DM. Following [39], here we do not consider the interaction operators $\hat{\mathcal{O}}_{2}$ and $\hat{\mathcal{O}}_{16}$ : the former is quadratic in $\hat{\boldsymbol{v}}^{\perp}$ (and the effective theory expansion in [39] is truncated at linear order in $\hat{\boldsymbol{v}}^{\perp}$ and second order in $\hat{\boldsymbol{q}}$ ) and the latter is a linear combination of $\hat{\mathcal{O}}_{12}$ and $\hat{\mathcal{O}}_{15}$.

$m_{\mathrm{DM}}=50 \mathrm{GeV}$ (the benchmark value used in our calculation), we find that it is always possible to identify a leading operator among those generated from a given simplified model in the non-relativistic limit.

\section{$3 \quad$ Statistical methods}

We compute exclusion limits and discovery regions (or sensitivity projections) using the profile likelihood ratio method [49]. In the former case, we compare the background plus signal hypothesis, $H_{1}$, with the background only hypothesis, $H_{0}$, computing the significance with which a point in parameter space can be excluded. In the latter case, we test the null hypothesis $H_{0}$ against the alternative $H_{1}$, computing the significance with which a point in parameter space can be observed. In both cases, we obtain the significance, $Z$, from a profile likelihood ratio $\lambda$ and the test statistic $q=-2 \ln \lambda$, using standard asymptotic formulae from [49]

$$
Z \simeq \sqrt{q}
$$

The significance is also related to the $p$-value, i.e. $Z=\Phi^{-1}(1-p)$, where $\Phi^{-1}$ is the quantile of a Gaussian distribution with mean 0 and variance 1 . For example, standard $95 \%$ confidence level (C.L.) exclusion limits correspond to a $p$-value of 0.05 and a significance of 1.64. 
The exact form of the profile likelihood ratio depends on whether we calculate discovery regions or exclusion limits. For exclusion limits, the profile likelihood ratio takes the following form

$$
\lambda=\frac{\mathscr{L}(\boldsymbol{s}, \widehat{\boldsymbol{\theta}})}{\mathscr{L}(\mathbf{0}, \widehat{\hat{\boldsymbol{\theta}}})},
$$

where the likelihood function, $\mathscr{L}$, is defined below. For discovery regions, the profile likelihood ratio is given by

$$
\lambda=\frac{\mathscr{L}(\mathbf{0}, \widehat{\widehat{\boldsymbol{\theta}}})}{\mathscr{L}(\boldsymbol{s}, \widehat{\boldsymbol{\theta}})} .
$$

Here, the likelihood function for finding a given signal $s=\left\{s_{1}, \ldots, s_{N}\right\}$ over a background $\boldsymbol{b}=\left\{b_{1}, \ldots, b_{N}\right\}$ for a dataset $\boldsymbol{n}=\left(n_{1}, \ldots, n_{N}\right)$ is defined as the product of $N$ Poisson distributions

$$
\mathscr{L}(\boldsymbol{s}, \boldsymbol{\theta})=\prod_{i=1}^{N} \frac{\left(s_{i}+b_{i}(\boldsymbol{\theta})\right)^{n_{i}}}{n_{i} !} \mathrm{e}^{-\left[s_{i}+b_{i}(\boldsymbol{\theta})\right]},
$$

where $N$ is the number of bins in the dijet invariant mass, $s_{i}$ the number of signal events in the $i$-th bin, $b_{i}$ the number of background events in the same bin, and $\boldsymbol{\theta}$ a set of nuisance parameters, i.e. the background model parameters from [50], in our case. ${ }^{3}$ In the definition(s) of $\lambda, \widehat{\boldsymbol{\theta}}(\widehat{\boldsymbol{\theta}})$ is the set of nuisance parameters maximizing the likelihood function for the given signal $\boldsymbol{s}(\mathbf{0})$. Maximising $\mathscr{L}$ with respect to $\boldsymbol{\theta}$ to find $\widehat{\boldsymbol{\theta}}$ or $\widehat{\boldsymbol{\theta}}$ at each point in parameter space, we exclude a window around the mediator mass in the dijet invariant mass spectrum. See [38] for further details.

Computing exclusion limits, we evaluate $Z$ for $n_{i}=n_{i}^{\mathrm{CMS}}$, where $n_{i}^{\mathrm{CMS}}$ is the number of observed dijet events at CMS in the $i$-th dijet mass bin. Computing $Z$ for discovery regions, we use the dataset $n_{i}=b_{i}\left(\boldsymbol{\theta}_{\mathrm{bf}}\right)+s_{i}$, where $\boldsymbol{\theta}_{\mathrm{bf}}$ is the value of $\boldsymbol{\theta}$ that maximises $\mathscr{L}(\mathbf{0}, \boldsymbol{\theta})$ for $n_{i}=n_{i}^{\mathrm{CMS}}$.

\section{Analysis}

In this section we investigate the impact of a XENONnT signal on the interpretation of current dijet searches at the LHC, and on the prospects for dijet signal discovery at the HL-LHC.

Let us start by describing our assumptions about the hypothesised XENONnT signal. We assume that XENONnT with an exposure of 20 ton $\times$ year has detected 150 nuclear recoil events due to DM-nucleus scattering. Roughly, this number of signal events corresponds to DM models lying just below current XENON1T limits. We consider an idealised version of the XENONnT detector with infinite energy resolution, an energy threshold of $5 \mathrm{keV}$, and

\footnotetext{
${ }^{3}$ For each parameter point independently, we maximise the likelihood over all invariant mass bins for a given $n_{i}$ (excluding a window around the mediator mass). The obtained model parameters include the systematic uncertainties on the background. The statistical uncertainties are accounted for by assuming a Poisson distribution of the events bin-by-bin around their expectation value.
} 


\begin{tabular}{|lcccc|}
\hline Spin $0 \mathrm{DM}$ & Op. & $g_{q}$ & $g_{\mathrm{DM}}$ & $M_{\text {eff }}[\mathrm{GeV}]$ \\
$(S \otimes S)_{0}$ & 1 & $h_{1}$ & $g_{1}$ & 14600 \\
$(S \otimes i \partial)_{0}$ & 1 & $h_{3}$ & $g_{4}$ & 10300 \\
\hline Spin $1 / 2 \mathrm{DM}$ & Op. & $g_{q}$ & $g_{\mathrm{DM}}$ & $M_{\text {eff }}[\mathrm{GeV}]$ \\
$(S \otimes S)_{1 / 2}$ & 1 & $h_{1}$ & $\lambda_{1}$ & 14600 \\
$(V \otimes V)_{1 / 2}$ & 1 & $h_{3}$ & $\lambda_{3}$ & 7260 \\
$(A \otimes A)_{1 / 2}$ & 4 & $h_{4}$ & $\lambda_{4}$ & 147 \\
$(V \otimes A)_{1 / 2}$ & 8 & $h_{3}$ & $\lambda_{4}$ & 225 \\
$(S \otimes P S)_{1 / 2}$ & 11 & $h_{1}$ & $\lambda_{2}$ & 352 \\
\hline $\mathrm{Spin} 1 \mathrm{DM}$ & $\mathrm{Op}$. & $g_{q}$ & $g_{\mathrm{DM}}$ & $M_{\text {eff }}[\mathrm{GeV}]$ \\
$(S \otimes S)_{1}$ & 1 & $h_{1}$ & $b_{1}$ & 14600 \\
$(V \otimes i \partial)_{1}$ & 1 & $h_{3}$ & $b_{5}$ & 10300 \\
\hline
\end{tabular}

Table 3. Benchmark points producing 150 signal events in an idealised version of XENONnT for $m_{\chi}=50 \mathrm{GeV}$ [28]. Consistently with [28], in the case of spin $1 \mathrm{DM}$ we do not consider the contribution to $M_{\text {eff }}$ from effective operators that depend on the symmetric combination of polarisation vectors denoted by $\mathcal{S}$. We omitted models that are not compatible with a dijet signal in the relevant parameter space.

$100 \%$ detector efficiency. ${ }^{4}$ To obtain the number of expected signal events at XENONnT, we follow [29] and integrate the differential rate of nuclear recoil events in eq. (2.3) in the 5 to $45 \mathrm{keV}$ range using DMFormFactor [46]. The total number of events is then obtained by multiplying the result by a 20 ton $\times$ year exposure. Direct detection experiments are not sensitive to the individual parameters of the simplified models, but only to the DM mass $m_{\mathrm{DM}}$ and the effective mediator mass

$$
M_{\mathrm{eff}} \equiv \frac{m_{\mathrm{med}}}{\left(g_{q} / 0.1\right)\left(g_{\mathrm{DM}} / 0.1\right)}
$$

Note that we chose to normalize the coupling constants to $g_{q}=g_{\mathrm{DM}}=0.1$, corresponding to typical values for weak couplings, in the definition of $M_{\mathrm{eff}}$.

For the simplified models in eq. (2.1), table 3 shows the values of $M_{\text {eff }}$ required to produce 150 signal events at an idealised version of XENONnT. Notice that a signal at XENONnT would constrain $M_{\text {eff }}$ univocally, with an associated relative uncertainty of about $20 \%$ [30] that would be negligible compared to astrophysical uncertainties. Furthermore, experimental errors on the reconstructed value of $m_{\mathrm{DM}}$ are also expected to be negli-

\footnotetext{
${ }^{4}$ The detector deficiencies and resolution of XENON will have a minor impact on $M_{\text {eff }}$, in particular compared to astrophysical uncertainties as discussed in more detail in ref. [28]. We use the 150 signal events in the idealized version of XENON as a benchmark. A more realistic treatment of the detector would lead to less, but still $\mathcal{O}(100)$, signal events for a given benchmark point. Furthermore, including detector efficiencies and resolution would not have significant consequences for comparing the impact of a direct detection signal in dijet searches for different models.
} 
gible in this setup, ${ }^{5}$ and we therefore set $m_{\mathrm{DM}}$ to its benchmark value, i.e. $m_{\mathrm{DM}}=50 \mathrm{GeV}$. In addition to the constraints on $M_{\mathrm{eff}}$ and $m_{\mathrm{DM}}$ from the detection of 150 signal events at XENONnT, we also require perturbative couplings $\left|g_{\mathrm{DM}}\right|<\sqrt{4 \pi}$ and $\left|g_{q}\right|<\sqrt{4 \pi}$. Finally, we assume universal quark couplings $g_{u}=g_{d}=g_{s}=g_{c}=g_{b}=g_{t} \equiv g_{q}$, and negligible coupling of the mediator to leptons, i.e. $g_{\ell} \simeq 0$, in agreement with current searches for resonances in dilepton final states at the LHC [52]. Having described our assumptions about the hypothesised XENONnT signal, we now investigate its impact on the interpretation of current dijet searches at the LHC (section 4.1), and on the prospects for dijet signal discovery at the HL-LHC (section 4.2).

\subsection{Impact of a XENONnT signal on LHC dijet exclusion limits}

For the benchmark parameters which would give rise to 150 signal events in XENONnT, we calculate $95 \%$ C.L. exclusion limits on the mediator's coupling to quarks, $g_{q}$, from current searches for resonances in dijet final states at the LHC. Because we fixed the $\mathrm{DM}$ mass to $m_{\mathrm{DM}}=50 \mathrm{GeV}$ (assuming perfect mass reconstruction), our XENONnT analysis described above yields a surface of parameter points in the space spanned by $\left\{m_{\text {med }}, g_{q}, g_{\mathrm{DM}}\right\}$, defined by the respective values of $M_{\text {eff }}$ reported in table 3 . On this surface, the mediator's coupling to DM, $g_{\mathrm{DM}}$, is a function of $m_{\text {med }}$ and $g_{q}$. Geometrically, the function $g_{\mathrm{DM}}=g_{\mathrm{DM}}\left(m_{\text {med }}, g_{q}\right)$ can be obtained by projecting the surface defined by $M_{\text {eff }}$ to the $m_{\text {med }}-g_{q}$ plane. In practice, for each benchmark point in table 3 we obtain $g_{\mathrm{DM}}$ by solving eq. (4.1) for $g_{\mathrm{DM}}$ at each point in the $\left(m_{\text {med }}, g_{q}\right)$ plane.

To calculate the $95 \%$ C.L. exclusion limits on $g_{q}\left(m_{\text {med }}\right)$ arising from resonant dijet searches at the LHC and a signal at XENONnT, we use the profile likelihood ratio method outlined in section 3. For each simplified model, we simulate the corresponding dijet invariant mass spectrum using the chain of numerical programmes described in section 2 on a grid in $\left(m_{\text {med }}, g_{q}\right)$, setting $g_{\mathrm{DM}}$ to the value obtained from the $M_{\text {eff }}$ constraint at each point and fixing $m_{\mathrm{DM}}=50 \mathrm{GeV}$. After simulating the dijet mass spectrum, we integrate it to obtain the number of expected dijet events, $s_{i}$, in bins of dijet invariant mass labeled by the integer $i$ and of variable width, as in the high-mass search for narrow resonances in dijet final states performed by CMS [50]. Following [50], we assume an integrated luminosity of $36 \mathrm{fb}^{-1}$, a centre-of-mass energy of $\sqrt{s}=13 \mathrm{TeV}$, and focus on the 1.6-3.9 TeV range for the dijet invariant mass. For models where 150 events in XENONnT arise for relatively large effective mediator masses, e.g. $M_{\text {eff }}=1.46 \mathrm{TeV}$ for $(S \otimes S)_{1 / 2}$, dijet searches at the LHC constrain regions of parameter space where $g_{q}$ is sizable and $g_{\mathrm{DM}} \ll g_{q}$ (with typical values of $\left.g_{\mathrm{DM}} \sim \mathcal{O}\left(10^{-3}\right)\right)$. Then, the mediator predominately decays into pairs of quarks. For models with much smaller $M_{\text {eff }}$ corresponding to 150 signal events in XENONnT, e.g. $M_{\text {eff }}=147 \mathrm{GeV}$ for $(A \otimes A)_{1 / 2}$, dijet searches constrain regions of parameter space where $g_{\mathrm{DM}} \gtrsim g_{q}$ (with typical values close to the perturbativity bound, $g_{\mathrm{DM}}=\sqrt{4 \pi}$ ), and hence the branching ratio of the mediator into pairs of quarks is suppressed.

\footnotetext{
${ }^{5}$ This holds as long as the mass of the DM candidate is not much heavier than the mass of a xenon nucleus, the target in XENONnT, see e.g. ref. [51].
} 


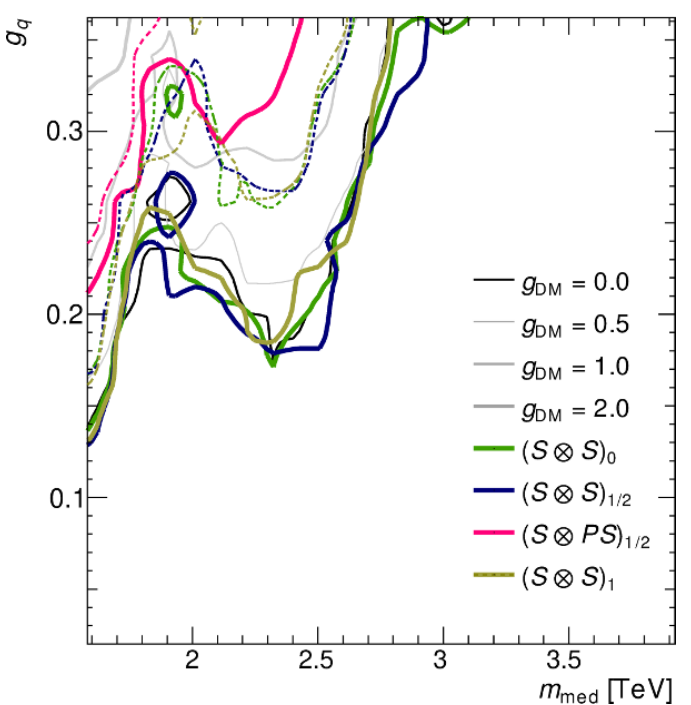

(a)

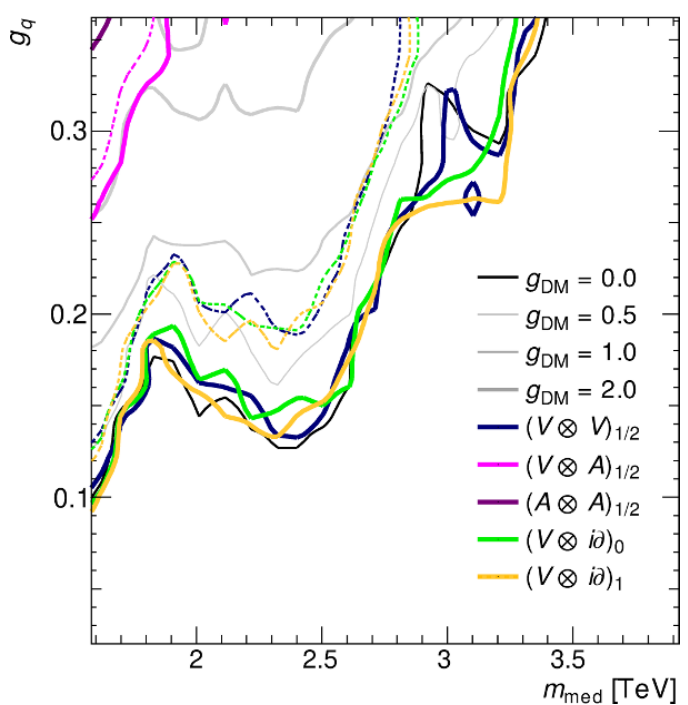

(b)

Figure 1. 95\% C.L. $(3 \sigma)$ exclusion limits on $g_{q}$ from the null result of present searches for narrow resonances in dijet final states at the LHC obtained by setting $m_{\mathrm{DM}}=50 \mathrm{GeV}$ and $g_{\mathrm{DM}}$ to the value required by the detection of 150 signal events at XENONnT indicated by solid (dotted) coloured lines. Exclusion limits are presented for simplified models with a scalar mediator (a) and for models with a vector mediator (b). We show only the models that are not yet fully excluded by dijet searches in the relevant parameter range. In both panels we used data from a CMS search for narrow resonances in final states involving a dijet corresponding to an integrated luminosity of $36 \mathrm{fb}^{-1}$. For models $(S \otimes S)_{1 / 2}$ (a) and $(V \otimes V)_{1 / 2}$ (b), the figure also shows $95 \%$ C.L. exclusion limits obtained by setting $g_{\mathrm{DM}}$ to XENONnT-independent values (grey lines).

Figure 1 shows the impact that the detection of 150 signal events at XENONnT would have on the $95 \%$ C.L. exclusion limits on $g_{q}$ from the null result of present searches for narrow resonances in dijet final states at the LHC. The left panel refers to simplified models with scalar or pseudo-scalar mediators, whereas the right panel corresponds to simplified models with vector or pseudo-vector mediators. In figure 1 we report exclusion limits only for a subset of the simplified models and corresponding benchmark points in appendix A. For the models not shown, the benchmark points would correspond to $95 \%$ C.L. exclusion limits extending to regions in parameter space where coupling constants are non-perturbative. Specifically, this applies to benchmark points where $M_{\text {eff }} \ll m_{\text {med }}$. In figure 1 we display all the models for which combinations of parameters which would give rise to 150 signal events in XENONnT are not yet excluded by LHC dijet results; labeled according to the notation introduced in eq. (2.1). For the model $(S \otimes S)_{1 / 2}$ in the left panel - fermionic DM and scalar mediator - and the model $(V \otimes V)_{1 / 2}$ in the right panel fermionic DM and vector mediator — we also show 95\% C.L. exclusion limits obtained using values for $g_{\mathrm{DM}}$ which are not related to constraints on $M_{\text {eff }}$ from the detection of 150 signal events at XENONnT (grey curves). For these two models, 150 signal events at XENONnT require $M_{\text {eff }} \gg m_{\text {med }}$, which implies $g_{\mathrm{DM}} \simeq 0$. This explains why for models $(S \otimes S)_{1 / 2}$ and $(V \otimes V)_{1 / 2} 95 \%$ C.L. exclusion limits computed assuming 150 signal events at XENONnT 


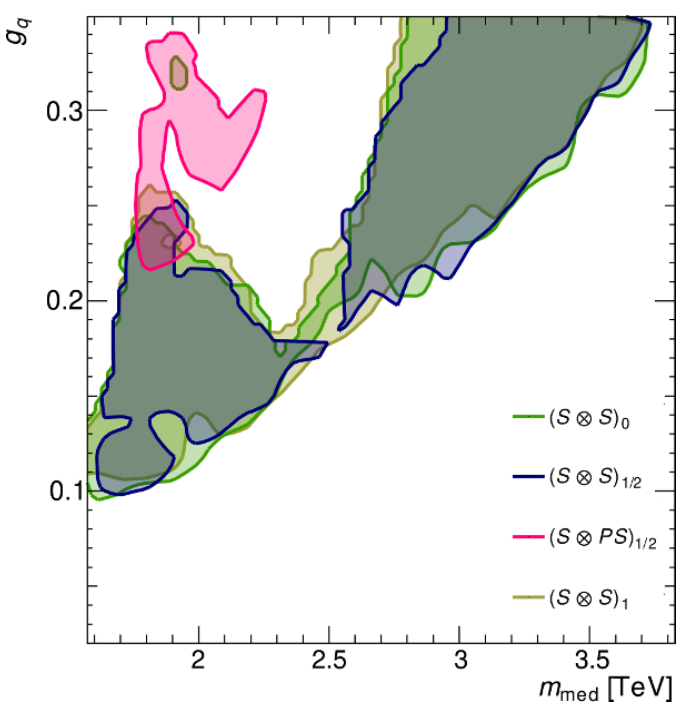

(a)

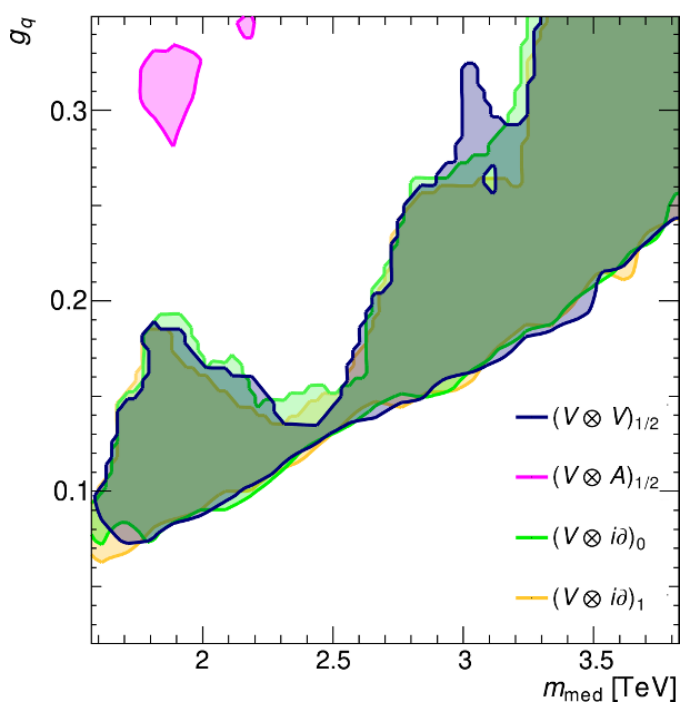

(b)

Figure 2. Regions in the $\left(m_{\text {med }}, g_{q}\right)$ plane where a narrow resonance could be discovered with $Z \geq 5 \sigma$ C.L. in dijet final states at the HL-LHC that are at the same time compatible with the detection of 150 signal events at XENONnT. The lower boundary of the region for each model is the smallest coupling $g_{q}\left(m_{\text {med }}\right)$ for which we would expect a $5 \sigma$ discovery at the HL-LHC, while the upper boundary is given by the current $95 \%$ C.L. exclusion limits from $36 \mathrm{fb}^{-1}$ of CMS data. The left panel (a) corresponds to simplified models with a scalar mediator, while the right panel (b) refers to models with a vector mediator.

or setting $g_{\mathrm{DM}}=0$ are close to each other. On the other hand, while exclusion limits in figure 1 depend only indirectly on $g_{\mathrm{DM}}$ via the total mediator decay width and branching ratio into quarks, a large coupling to DM can significantly reduce the branching ratio into quarks, and therefore lead to significantly weaker exclusion limits on $g_{q}$.

\subsection{Impact of a XENONnT signal on LHC dijet $5 \sigma$ discovery contours}

In this section, we investigate the impact of a XENONnT signal on the prospects for dijet signal discovery at the HL-LHC. We use the profile likelihood ratio method outlined in section 3 to identify the contours in the $\left(m_{\text {med }}, g_{q}\right)$ plane where simultaneously: 1$)$ a narrow resonance in dijet final states at the HL-LHC could be discovered with a statistical significance of $5 \sigma ; 2) 150$ signal events are expected at XENONnT. As before, we set $m_{\mathrm{DM}}$ to $50 \mathrm{GeV}$ and extract $g_{\mathrm{DM}}$ from $M_{\text {eff }}$ using the XENONnT input. Concerning dijet signal and background calculation, as well as our choice of likelihood function and dataset, we proceed as described in section 3.

Figure 2 shows the regions in the $\left(m_{\text {med }}, g_{q}\right)$ plane where a dijet signal could be discovered at the HL-LHC with a statistical significance larger than or equal to $5 \sigma$, and which are at the same time compatible with the detection of 150 signal events at XENONnT. Regions with different colours correspond to distinct simplified models in eq. (2.1). In each region, the lower boundary $g_{q}^{\min }\left(m_{\text {med }}\right)$ is the smallest coupling $g_{q}$ for which the corresponding model could be discovered with a significance of $5 \sigma$ in dijet searches at the HL-LHC. The 
upper boundary $g_{q}^{\max }\left(m_{\text {med }}\right)$ is given by the $95 \%$ C.L, exclusion limits from $36 \mathrm{fb}^{-1}$ of data discussed in section 4.1, cf. figure 1. The left panel corresponds to simplified models with a scalar or pseudo-scalar mediator, while the right panel refers to models with vector or pseudo-vector mediators. We use the labels introduced in the previous sections. Models that do not appear in figure 2 are not compatible with the simultaneous discovery of 150 signal events at XENONnT and the $5 \sigma$ detection of a dijet signal at the HL-LHC. Note that in case of no signal discovery the $5 \sigma$ lines in figure 2 would roughly correspond to the corresponding HL-LHC exclusion limits with the same significance.

Interestingly, we find that only a subset of models would actually be compatible with the simultaneous detection of a signal at XENONnT and at the HL-LHC. Furthermore, we find that some of the models in figure 2 can potentially be distinguished in dijet searches, since the mediator mass can approximately be reconstructed from an analysis of the dijet invariant mass at the HL-LHC. For example, the discovery of a dijet signal for a mediator mass $m_{\text {med }} \gtrsim 2.5 \mathrm{TeV}$ would exclude the models $(S \otimes P S)_{1 / 2}$ and $(V \otimes A)_{1 / 2}$, leaving only models with $\hat{\mathcal{O}}_{1}$ as leading non-relativistic operator for DM-nucleon interactions. On the other hand, the discovery of a dijet signal at lower mediator masses would also be compatible with models $(S \otimes P S)_{1 / 2}$ and $(V \otimes A)_{1 / 2}$, which in the non-relativistic limit generate the interaction operators $\hat{\mathcal{O}}_{11}$ and $\hat{\mathcal{O}}_{8}$, respectively. Note, that the regions shown in figure 2 do not take into account limits on simplified models which can be obtained from the preliminary results published by the CMS collaborations from $78 \mathrm{fb}^{-1}$ of data [25] presented in [38]. These results rule out all of the regions for the models $(S \otimes P S)_{1 / 2}$ and $(V \otimes A)_{1 / 2}$ shown in figure 2 at $95 \%$ C.L. However, a $5 \sigma$ discovery could still be made at the HL-LHC for such models if one loosens the assumption of 150 signal events being produced at XENONnT. Regarding the models with couplings $\left[h_{3}, \operatorname{Re}\left(b_{7}\right)\right]$ and $\left[h_{3}, \operatorname{Im}\left(b_{7}\right)\right]$, a spin 1 mediator with a spin $1 \mathrm{DM}$ candidate, cf. eq. (A.6), we did not compute them explicitly. The benchmark value for the effective mediator mass giving rise to 150 events in XENONnT is $M_{\text {eff }} \sim 200 \mathrm{GeV}$, thus, one would expect similar regions as in the $(V \otimes A)_{1 / 2}$ scenario. However, the partial width for a spin 1 mediator decaying via $b_{7}$ is enhanced by a factor $\sim\left(m_{\text {med }} / m_{\mathrm{DM}}\right)^{2}$ with respect to a decay via $\lambda_{3}$ in the $(V \otimes A)_{1 / 2}$ model [30]. Thus, the branching ratio into quarks is suppressed and we do not expect a significant chance to discover such a scenario at the HL-LHC.

As an aside comment, we mention here that $g_{q}$ could in principle be inferred from the measurement of the mediator decay width, assuming that $m_{\text {med }}$ and $M_{\text {eff }}$ are both known. However, this would require a very accurate measurement of the dijet invariant mass spectrum to extract the mediator decay width from data collected at the HL-LHC. Most likely, the number of signal events recorded in the initial stages of the HL-LHC would not suffice to reconstruct the invariant mass spectrum with the precision required to indirectly infer $g_{q}$.

In the left panel of figure 3 we compare the two models $(S \otimes S)_{0}$ and $(V \otimes V)_{1 / 2}$ in more detail. We find that these models predict partly non overlapping contours in the $\left(m_{\text {med }}, g_{q}\right)$ plane. This is an interesting result, since it shows that models generating $\hat{\mathcal{O}}_{1}$ as leading non-relativistic operator can in principle be discriminated if a dijet signal is 


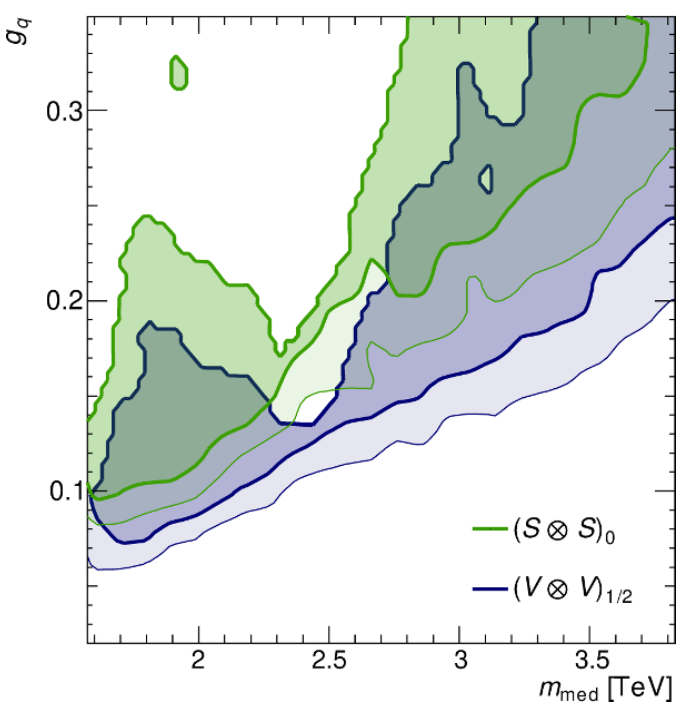

(a)

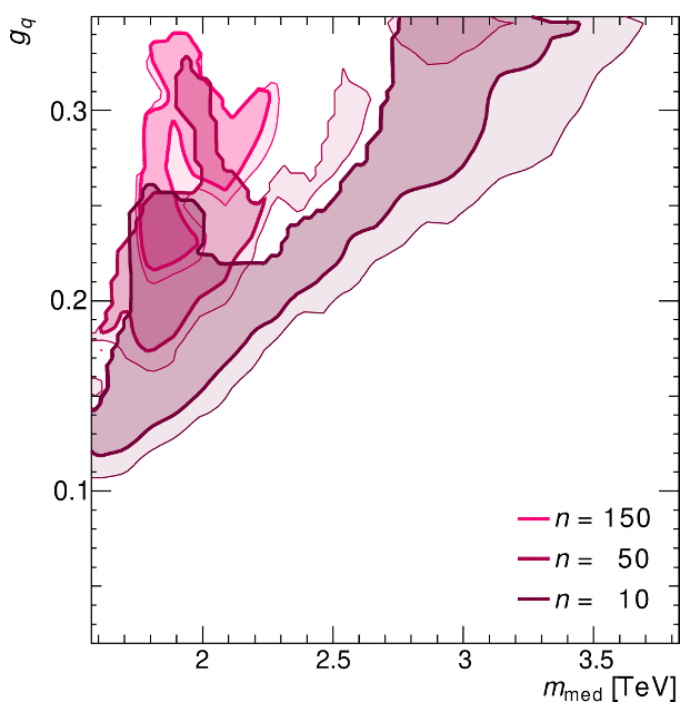

(b)

Figure 3. The left panel (a) is the same as figure 2, but now focusing on the models $(S \otimes S)_{0}$ and $(V \otimes V)_{1 / 2}$. Both models generate $\hat{\mathcal{O}}_{1}$ as a leading non-relativistic operator for DM-nucleon interactions. The thick (thin) outline corresponds to a $5 \sigma(3 \sigma)$ discovery. Discovery contours are partly non overlapping. In the right panel (b) we show how the regions where dijets searches could make a discovery in the $(S \otimes P S)_{1 / 2}$ model if instead of $n=150$ only $n=50$ or $n=10$ events would be observed at XENONnT.

observed at the HL-LHC. ${ }^{6}$ This is in contrast with what we found investigating the impact of a XENONnT signal on LHC monojet searches [28]. In that work, we found that models generating $\hat{\mathcal{O}}_{1}$ as leading operator in the non-relativistic limit are not observable in monojet searches at the LHC if the model parameters are such that $\mathcal{O}(100)$ signal events would be observed at XENONnT [28].

Let us now investigate the dependence of our results on the number of signal events observed at XENONnT, $n$. Figure 3, right panel, shows how discovery regions change if instead of $n=150$ signal events, only $n=50$ or $n=10$ events are observed at XENONnT. As an example, we show results for the model $(S \otimes P S)_{1 / 2}$. Note that the effective mass reconstructed from XENONnT scales with the number of events as $M_{\text {eff }} \sim n^{-4}$. For a fixed combination of parameters $\left(m_{\text {med }}, g_{q}\right)$ this implies the scaling $g_{\mathrm{DM}} \sim \sqrt{n}$. A larger number of events at XENONnT therefore implies a larger partial decay width of the mediator into DM for a given $g_{q}$ and mediator mass, which weakens the significance of a potential dijet signal. Fewer events at XENONnT instead lead to the predominant decay into SM particles leading to a stronger dijet signal in comparison, which is limited by the ideal case where $g_{\mathrm{DM}} \cong 0$ and the mediator decays exclusively into SM particles.

\footnotetext{
${ }^{6}$ The island like structures in figure 3 as well as the $3 \sigma$ C.L exclusion limit shown in figure 1 (dotted lines) indicate that the uncertainties in the upper boundary of the region (corresponding to the current $95 \%$ C.L exclusion limit) are larger than the separation of the models. If we focus on the lower boundary, however, we see that there is a remaining region where a signal could only be discovered with $5 \sigma$ significance for the model $(V \otimes V)_{1 / 2}$ and not for $(S \otimes S)_{0}$ thus allowing in principle for a separation of the models in part of the parameter space.
} 
As a consequence, the exclusion limits as well as the $5 \sigma$ discovery contour move towards higher masses and smaller quark couplings. Furthermore, the overall region where a discovery at the HL-LHC is possible becomes larger. We expect a similar behaviour for the model $(V \otimes A)_{1 / 2}$. For the remaining models shown in figure 2, the mediator's decay width is dominated by the partial width corresponding to decays into quarks. Therefore, a smaller number of events observed at XENONnT implying a smaller coupling $g_{\mathrm{DM}}$ for fixed $g_{q}$ and $m_{\text {med }}$ would have virtually no impact on the mediator's decay width and branching ratios. Thus, the regions in which such models could give rise to a $5 \sigma$ discovery at the HL-LHC are nearly independent of the number of events observed at XENONnT.

Let us also qualitatively investigate what impact a DM mass different from $m_{\mathrm{DM}}=50 \mathrm{GeV}$, would have on our results. The detector can resolve dark matter masses up to about $100 \mathrm{GeV}$ reasonably well, see, e.g., refs. [30, 51]. For larger masses the direct detection signal becomes largely independent of $m_{\mathrm{DM}}$. Due to this degeneracy, XENONnT is not expected to achieve an accurate reconstruction of dark matter masses above $\sim 100 \mathrm{GeV}$. We therefore compare the mass $m_{\mathrm{DM}}=50 \mathrm{GeV}$, where XENONnT is most sensitive, with $m_{\mathrm{DM}}=100 \mathrm{GeV}$. We restrict our discussion to the models $(S \otimes P S)_{1 / 2}$ and $(V \otimes A)_{1 / 2}$ for which prospects at the HL-LHC depend strongly on the number of events observed at XENONnT, $n$. Naively, one would assume that the dijet production cross section depends on the DM mass via the branching ratios of the mediator. However, the dependence on the mediator mass, $m_{\text {med }}$, is much stronger, yielding virtually unchanged discovery regions when assuming $m_{\mathrm{DM}}=100 \mathrm{GeV}$ instead of $m_{\text {med }}=50 \mathrm{GeV}$. On the other hand, $\mathrm{DM}$ direct detection experiments using xenon as target material can probe the smallest WIMP-nucleon scattering cross sections for DM masses of $m \sim 50 \mathrm{GeV}$. Thus, observing 150 events at XENONnT corresponds to larger WIMP-nucleus cross sections, and in turn smaller $M_{\text {eff }}$, for larger $m_{\mathrm{DM}}$. Since smaller $M_{\mathrm{eff}}$ correspond to larger $g_{\mathrm{DM}}$ for fixed values of $m_{\text {med }}$ and $g_{q}$, increasing $m_{\mathrm{DM}}$ to values larger than $50 \mathrm{GeV}$ has the opposite effect as a smaller number of events observed at XENONnT discussed above. Similar to that case, the discovery regions presented for the other models considered here are expected to remain approximately unchanged when for example assuming $m_{\mathrm{DM}}=100 \mathrm{GeV}$ instead of $m_{\text {med }}=50 \mathrm{GeV}$.

Finally, we would like to stress that the two models $(S \otimes P S)_{1 / 2}$ and $(V \otimes A)_{1 / 2}$ can simultaneously be observed in monojet [28] and dijet searches at the LHC (see figure 2). For model $(S \otimes P S)_{1 / 2}$, a dijet signal is only observable close to $m_{\text {med }} \approx 2 \mathrm{TeV}, g_{q} \approx 0.3$ and $g_{\mathrm{DM}} \approx 1$, as it can be inferred from figure 2. Interestingly, these models also generate non-relativistic operators for DM-nucleon interactions, $\hat{\mathcal{O}}_{11}$ and $\hat{\mathcal{O}}_{8}$, respectively, which can statistically be discriminated from an analysis of the associated nuclear recoil energy spectra [28]. These models can therefore be very effectively constrained from a combined analysis of LHC and XENONnT data.

\section{Conclusion}

In this work we have investigated the impact that a signal at XENONnT would have on the interpretation of current dijet searches at the LHC, and on the prospects for dijet 
signal discovery at the High-Luminosity LHC in the framework of simplified models. In the analysis, we have focused on simplified models where DM can have spin $0,1 / 2$ or 1 , and primarily interacts with quarks through the exchange of scalar, pseudo-scalar, vector, or pseudo-vector mediators.

Assessing the impact of a XENONnT signal on the interpretation of current dijet searches at the LHC, we have calculated 95\% C.L. exclusion limits on the coupling constant associated with the mediator-quark-quark vertex, $g_{q}$, as a function of the mediator mass, $m_{\text {med }}$, from the null result of current searches for resonances in dijet final states at the LHC. We have performed this calculation for the simplified models described above (and in greater detail in appendix $\mathrm{A}$ ), setting $m_{\mathrm{DM}}$ to the benchmark value of $50 \mathrm{GeV}$, and taking into account the constraint on the effective mediator mass $M_{\text {eff }}$ [defined in eq. (4.1)] arising from the detection of 150 signal events at XENONnT. The 95\% C.L. exclusion limits on $g_{q}$ presented here have been calculated using the standard profile likelihood method [49]. We have found that for models for which a XENONnT signal implies $M_{\text {eff }} \ll m_{\text {med }}$ (in the range of $m_{\text {med }}$ values that we have considered), 95\% C.L. exclusion limits extend to regions in parameter space where coupling constants are non-perturbative, and therefore become trivial, because of DM detection at XENONnT. At the same time, we have found that models for which 150 signal events at XENONnT require $M_{\text {eff }} \gg m_{\text {med }}$ are characterised by the constraint $g_{\mathrm{DM}} \simeq 0$. In general, we have found that while exclusion limits in the $\left(m_{\text {med }}, g_{q}\right)$ plane depend only indirectly on $g_{\mathrm{DM}}$ via the total mediator decay width and branching ratio into quarks, a large coupling to DM [i.e. $g_{\mathrm{DM}} \sim \mathcal{O}(1)$ ] can significantly reduce the branching ratio into quarks, and therefore lead to significantly weaker exclusion limits on $g_{q}$.

Assessing the impact of a XENONnT signal on the prospects for dijet signal discovery at the HL-LHC, we have identified the contours in the $\left(m_{\text {med }}, g_{q}\right)$ plane where a narrow resonance could be discovered with a statistical significance of $5 \sigma$ in dijet final states at the HL-LHC, and which are at the same time compatible with the detection of 150 signal events at XENONnT. Interestingly, we have found that only a subset of the simplified models in appendix A would actually be compatible with the simultaneous detection of a signal at XENONnT and at the HL-LHC. We have also found that some of the models for which the two signals are compatible can potentially be distinguished if the mediator mass is approximately reconstructed from an analysis of the dijet invariant mass at the HL-LHC. Finally, we have found that models generating $\hat{\mathcal{O}}_{1}$ as the leading non-relativistic operator (i.e. canonical spin independent interactions) can in principle be discriminated if a dijet signal is observed at the HL-LHC. Notably, in a previous work [28] we have found that the same models cannot be discriminated by combining a signal at XENONnT with the LHC monojet searches.

Ultimately, our work has explored a new aspect of the well-known complementarity between DM searches at direct detection experiments and at the LHC. The results obtained in this study will be especially useful if DM will be discovered at XENONnT, but the methods illustrated here can in principle be applied to other combinations of DM search experiments. 


\section{Acknowledgments}

We would like to thank Jan Conrad and Katherine Freese for their contribution to the early stages of this project. We are grateful to Caterina Doglioni and Felix Kahlhoefer for useful insights into how to model dijet signal and background events at the LHC. Finally, it is a pleasure to thank Kåre Fridell and Vanessa Zema for helpful discussions on nonrelativistic effective theories and simplified models for dark matter. This work is partly performed within the Swedish Consortium for Dark Matter Direct Detection (SweDCube), and was supported by the Knut and Alice Wallenberg Foundation (PI, Jan Conrad) and by the Vetenskapsrådet (Swedish Research Council) through contract No. 638-2013-8993 (PI, Katherine Freese).

\section{A Lagrangians for simplified dark matter models}

In this appendix we list the Lagrangians that we considered in the analyses of section 4.1 and section $4.2[28,29]$. Each Lagrangian listed here describe more than one simplified model. By construction, simplified models are characterised by DM particle mass, $m_{\mathrm{DM}}$, mediator mass $m_{\text {med }}$, and just two coupling constants: one for a quark-quark-mediator vertex, $g_{q}$, and one for a DM-DM-mediator vertex, $g_{\mathrm{DM}}$. There are no other interaction vertices in a simplified model. For example, the simplified model associated with fermionic $\mathrm{DM}$ of mass $m_{\chi}$ and vector mediator of mass $m_{G}$, has $g_{q}=h_{3}$ and $g_{\mathrm{DM}}=\lambda_{3}$ as only coupling constants different from zero. In all numerical applications, we assumed a universal quark-mediator coupling.

\section{A.1 Scalar dark matter $S$}

\section{Scalar and pseudoscalar mediator $\phi$ :}

$$
\begin{aligned}
\mathcal{L}_{S \phi q}= & \partial_{\mu} S^{\dagger} \partial^{\mu} S-m_{S}^{2} S^{\dagger} S-\frac{\lambda_{S}}{2}\left(S^{\dagger} S\right)^{2} \\
& +\frac{1}{2} \partial_{\mu} \phi \partial^{\mu} \phi-\frac{1}{2} m_{\phi}^{2} \phi^{2}-\frac{m_{\phi} \mu_{1}}{3} \phi^{3}-\frac{\mu_{2}}{4} \phi^{4} \\
& +\mathrm{i} \bar{q} \not D q-m_{q} \bar{q} q \\
& -g_{1} m_{S} S^{\dagger} S \phi-\frac{g_{2}}{2} S^{\dagger} S \phi^{2}-h_{1} \bar{q} q \phi-i h_{2} \bar{q} \gamma^{5} q \phi
\end{aligned}
$$

Here, $m_{S}$ plays the role of $m_{\mathrm{DM}}$ and $m_{\phi}$ that of $m_{\text {med }} \cdot \lambda_{S}$ is a dimensionless self-coupling of $S$ and the $\mu_{i}$ are dimensionless self-couplings of the mediator. For the purposes of this work, we set $\lambda_{S}=\mu_{1}=\mu_{2}=0$. The $g_{i}$ are dimensionless couplings between $\phi$ and $S$. The couplings $h_{i}$ between the mediators and quarks and the quark mass matrix $m_{q}$ should in general be understood as $(6 \times 6)$ matrices and the quarks fields as vectors $q=(u, d, c, s, t, b)$ in flavour space. Throughout this work we assume universal (diagonal) couplings of the mediators to quarks such that the $h_{i}$ can be treated as a single number. The quark mass matrix $m_{q}$ can be assumed to be diagonal. 


\section{Vector and axial-vector mediator $G_{\mu}$ :}

$$
\begin{aligned}
\mathcal{L}_{S G q}= & \partial_{\mu} S^{\dagger} \partial^{\mu} S-m_{S}^{2} S^{\dagger} S-\frac{\lambda_{S}}{2}\left(S^{\dagger} S\right)^{2} \\
& -\frac{1}{4} \mathcal{G}_{\mu \nu} \mathcal{G}^{\mu \nu}+\frac{1}{2} m_{G}^{2} G_{\mu} G^{\mu}-\frac{\lambda_{G}}{4}\left(G_{\mu} G^{\mu}\right)^{2} \\
& +i \bar{q} \not D q-m_{q} \bar{q} q \\
& -\frac{g_{3}}{2} S^{\dagger} S G_{\mu} G^{\mu}-i g_{4}\left(S^{\dagger} \partial_{\mu} S-\partial_{\mu} S^{\dagger} S\right) G^{\mu} \\
& -h_{3}\left(\bar{q} \gamma_{\mu} q\right) G^{\mu}-h_{4}\left(\bar{q} \gamma_{\mu} \gamma^{5} q\right) G^{\mu}
\end{aligned}
$$

Here, $\mathcal{G}_{\mu \nu}$ is the field strength tensor of $G_{\mu}, m_{G}$ plays the role of $m_{\text {med }}$, and $\lambda_{G}$ is a dimensionless self-coupling of $G_{\mu}$ which we set to zero for the purposes of this work. The $g_{i}$ are the dimensionless couplings of $S$ to $G_{\mu}$, and the $h_{i}$ are the couplings of $G_{\mu}$ to quarks. As before, the $h_{i}$ are in general $(6 \times 6)$ matrices in flavor space, but can be treated as single numbers for the universal quark coupling assumed here. The remaining parameter are as in eq. (A.1).

\section{A.2 Fermionic dark matter $\chi$}

\section{Scalar and pseudoscalar mediator $\phi:$}

$$
\begin{aligned}
\mathcal{L}_{\chi \phi q}= & \mathrm{i} \bar{\chi} \not D_{\chi}-m_{\chi} \bar{\chi} \chi \\
& +\frac{1}{2} \partial_{\mu} \phi \partial^{\mu} \phi-\frac{1}{2} m_{\phi}^{2} \phi^{2}-\frac{m_{\phi} \mu_{1}}{3} \phi^{3}-\frac{\mu_{2}}{4} \phi^{4} \\
& +\mathrm{i} \bar{q} \not \phi_{q}-m_{q} \bar{q} q \\
& -\lambda_{1} \phi \bar{\chi} \chi-i \lambda_{2} \phi \bar{\chi} \gamma^{5} \chi-h_{1} \phi \bar{q} q-i h_{2} \phi \bar{q} \gamma^{5} q .
\end{aligned}
$$

Here, $m_{\chi}$ plays the role of $m_{\mathrm{DM}}$. The $\lambda_{i}$ are the dimensionless couplings between $\phi$ and $\chi$. The remaining parameter are as in eq. (A.1).

\section{Vector and axial-vector mediator $G_{\mu}$ :}

$$
\begin{aligned}
\mathcal{L}_{\chi G q}= & \mathrm{i} \bar{\chi} \not D \chi-m_{\chi} \bar{\chi} \chi \\
& -\frac{1}{4} \mathcal{G}_{\mu \nu} \mathcal{G}^{\mu \nu}+\frac{1}{2} m_{G}^{2} G_{\mu} G^{\mu} \\
& +\mathrm{i} \bar{q} \not \supset q-m_{q} \bar{q} \\
& -\lambda_{3} \bar{\chi} \gamma^{\mu} \chi G_{\mu}-\lambda_{4} \bar{\chi} \gamma^{\mu} \gamma^{5} \chi G_{\mu} \\
& -h_{3} \bar{q} \gamma_{\mu} q G^{\mu}-h_{4} \bar{q} \gamma_{\mu} \gamma^{5} q G^{\mu} .
\end{aligned}
$$

Beyond the parameters appearing in eqs. (A.2) and (A.3), the $\lambda_{i}$ are the dimensionless couplings between $\chi$ and $G_{\mu}$. 


\section{A.3 Vector dark matter $\boldsymbol{X}_{\mu}$}

\section{Scalar and pseudoscalar mediator $\phi$ :}

$$
\begin{aligned}
\mathcal{L}_{X \phi q}= & -\frac{1}{2} \mathcal{X}_{\mu \nu}^{\dagger} \mathcal{X}^{\mu \nu}+m_{X}^{2} X_{\mu}^{\dagger} X^{\mu}-\frac{\lambda_{X}}{2}\left(X_{\mu}^{\dagger} X^{\mu}\right)^{2} \\
& +\frac{1}{2}\left(\partial_{\mu} \phi\right)^{2}-\frac{1}{2} m_{\phi}^{2} \phi^{2}-\frac{m_{\phi} \mu_{1}}{3} \phi^{3}-\frac{\mu_{2}}{4} \phi^{4} \\
& +\mathrm{i} \bar{q} \not D_{q}-m_{q} \bar{q} q \\
& -b_{1} m_{X} \phi X_{\mu}^{\dagger} X^{\mu}-\frac{b_{2}}{2} \phi^{2} X_{\mu}^{\dagger} X^{\mu} \\
& -h_{1} \phi \bar{q} q-i h_{2} \phi \bar{q} \gamma^{5} q .
\end{aligned}
$$

Here, $\mathcal{X}_{\mu \nu}$ is the field strength tensor of $X_{\mu}, m_{X}$ plays the role of $m_{\mathrm{DM}}$, and $\lambda_{X}$ a dimensionless self-coupling of $X_{\mu}$ which we set to zero for the purposes of this work. The $b_{i}$ are dimensionless couplings of $\phi$ to $X_{\mu}$. The remaining parameters are as in eq. (A.1).

\section{Vector and axial-vector mediator $G_{\mu}$ :}

$$
\begin{aligned}
\mathcal{L}_{X G q}= & -\frac{1}{2} \mathcal{X}_{\mu \nu}^{\dagger} \mathcal{X}^{\mu \nu}+m_{X}^{2} X_{\mu}^{\dagger} X^{\mu}-\frac{\lambda_{X}}{2}\left(X_{\mu}^{\dagger} X^{\mu}\right)^{2} \\
& -\frac{1}{4} \mathcal{G}_{\mu \nu} \mathcal{G}^{\mu \nu}+\frac{1}{2} m_{G}^{2} G_{\mu}^{2}-\frac{\lambda_{G}}{4}\left(G_{\mu} G^{\mu}\right)^{2} \\
& +i \bar{q} \not D_{q}-m_{q} \bar{q} q \\
& -\frac{b_{3}}{2} G_{\mu}^{2}\left(X_{\nu}^{\dagger} X^{\nu}\right)-\frac{b_{4}}{2}\left(G^{\mu} G^{\nu}\right)\left(X_{\mu}^{\dagger} X_{\nu}\right) \\
& -\left[i b_{5} X_{\nu}^{\dagger} \partial_{\mu} X^{\nu} G^{\mu}+b_{6} X_{\mu}^{\dagger} \partial^{\mu} X_{\nu} G^{\nu}\right. \\
& \left.+b_{7} \varepsilon_{\mu \nu \rho \sigma}\left(X^{\dagger \mu} \partial^{\nu} X^{\rho}\right) G^{\sigma}+\text { h.c. }\right] \\
& -h_{3} G_{\mu} \bar{q} \gamma^{\mu} q-h_{4} G_{\mu} \bar{q} \gamma^{\mu} \gamma^{5} q .
\end{aligned}
$$

Here, the $b_{i}$ are dimensionless couplings between $X_{\mu}$ and $G_{\mu}$. The remaining parameters are as in eqs. (A.2) and (A.5).

Open Access. This article is distributed under the terms of the Creative Commons Attribution License (CC-BY 4.0), which permits any use, distribution and reproduction in any medium, provided the original author(s) and source are credited.

\section{References}

[1] G. Bertone, D. Hooper and J. Silk, Particle dark matter: Evidence, candidates and constraints, Phys. Rept. 405 (2005) 279 [hep-ph/0404175] [INSPIRE].

[2] G. Bertone and D. Hooper, History of dark matter, Rev. Mod. Phys. 90 (2018) 045002 [arXiv: 1605.04909] [INSPIRE].

[3] G. Arcadi et al., The waning of the WIMP? A review of models, searches and constraints, Eur. Phys. J. C 78 (2018) 203 [arXiv:1703.07364] [INSPIRE]. 
[4] A. Drukier and L. Stodolsky, Principles and Applications of a Neutral Current Detector for Neutrino Physics and Astronomy, Phys. Rev. D 30 (1984) 2295 [INSPIRE].

[5] M.W. Goodman and E. Witten, Detectability of Certain Dark Matter Candidates, Phys. Rev. D 31 (1985) 3059 [INSPIRE].

[6] XENON collaboration, Dark Matter Search Results from a One Ton-Year Exposure of XENON1T, Phys. Rev. Lett. 121 (2018) 111302 [arXiv:1805.12562] [INSPIRE].

[7] LUX collaboration, Dark Matter Searches with LUX, in Proceedings, 52nd Rencontres de Moriond on Very High Energy Phenomena in the Universe, La Thuile, Italy, March 18-25, 2017, pp. 199-209 (2017) [arXiv: 1710.03572] [INSPIRE].

[8] PandaX-II collaboration, Dark Matter Results From 54-Ton-Day Exposure of PandaX-II Experiment, Phys. Rev. Lett. 119 (2017) 181302 [arXiv:1708.06917] [INSPIRE].

[9] XENON collaboration, Physics reach of the XENON1T dark matter experiment, JCAP 04 (2016) 027 [arXiv: 1512.07501] [INSPIRE].

[10] B.J. Mount et al., LUX-ZEPLIN (LZ) Technical Design Report, arXiv:1703.09144 [INSPIRE].

[11] PandaX collaboration, Dark matter direct search sensitivity of the PandaX-4T experiment, Sci. China Phys. Mech. Astron. 62 (2019) 31011 [arXiv: 1806.02229] [INSPIRE].

[12] LHC roadmap: according to MTP 2016-2020 V1, https://lhc-commissioning.web.cern.ch/lhc-commissioning/schedule/LHC\%20schedule\% 20beyond\%20LS1\%20MTP\%202015_Freddy_June2015.pdf.

[13] A. De Simone and T. Jacques, Simplified models vs. effective field theory approaches in dark matter searches, Eur. Phys. J. C 76 (2016) 367 [arXiv: 1603.08002] [INSPIRE].

[14] O. Buchmueller, M.J. Dolan and C. McCabe, Beyond Effective Field Theory for Dark Matter Searches at the LHC, JHEP 01 (2014) 025 [arXiv:1308.6799] [INSPIRE].

[15] J. Abdallah et al., Simplified Models for Dark Matter Searches at the LHC, Phys. Dark Univ. 9-10 (2015) 8 [arXiv:1506.03116] [INSPIRE].

[16] F. Kahlhoefer, K. Schmidt-Hoberg, T. Schwetz and S. Vogl, Implications of unitarity and gauge invariance for simplified dark matter models, JHEP 02 (2016) 016 [arXiv: 1510.02110] [INSPIRE].

[17] J. Ellis, M. Fairbairn and P. Tunney, Anomaly-Free Dark Matter Models are not so Simple, JHEP 08 (2017) 053 [arXiv: 1704.03850] [INSPIRE].

[18] CMS collaboration, Search for new physics in final states with an energetic jet or a hadronically decaying $W$ or $Z$ boson and transverse momentum imbalance at $\sqrt{s}=13 \mathrm{TeV}$, Phys. Rev. D 97 (2018) 092005 [arXiv:1712.02345] [INSPIRE].

[19] ATLAS collaboration, Search for dark matter and other new phenomena in events with an energetic jet and large missing transverse momentum using the ATLAS detector, JHEP 01 (2018) 126 [arXiv: 1711.03301] [INSPIRE].

[20] M. Chala, F. Kahlhoefer, M. McCullough, G. Nardini and K. Schmidt-Hoberg, Constraining Dark Sectors with Monojets and Dijets, JHEP 07 (2015) 089 [arXiv:1503.05916] [INSPIRE].

[21] D. Abercrombie et al., Dark Matter Benchmark Models for Early LHC Run-2 Searches: Report of the ATLAS/CMS Dark Matter Forum, arXiv:1507.00966 [INSPIRE]. 
[22] ATLAS collaboration, Search for new phenomena in dijet events using $37 \mathrm{fb}^{-1}$ of $p p$ collision data collected at $\sqrt{s}=13$ TeV with the ATLAS detector, Phys. Rev. D 96 (2017) 052004 [arXiv: 1703.09127] [INSPIRE].

[23] CMS collaboration, Searches for dijet resonances in pp collisions at $\sqrt{s}=13 \mathrm{TeV}$ using data collected in 2016, CMS-PAS-EXO-16-056.

[24] CMS collaboration, Search for narrow and broad dijet resonances in proton-proton collisions at $\sqrt{s}=13 \mathrm{TeV}$ and constraints on dark matter mediators and other new particles, JHEP 08 (2018) 130 [arXiv: 1806.00843] [inSPIRE].

[25] CMS collaboration, Searches for dijet resonances in pp collisions at $\sqrt{s}=13 \mathrm{TeV}$ using the 2016 and 2017 datasets, CMS-PAS-EXO-17-026.

[26] ATLAS collaboration, Search for dijet resonances in events with an isolated lepton using $\sqrt{s}=13 \mathrm{TeV}$ proton-proton collision data collected by the ATLAS detector, ATLAS-CONF-2018-015.

[27] E.A. Baltz, M. Battaglia, M.E. Peskin and T. Wizansky, Determination of dark matter properties at high-energy colliders, Phys. Rev. D 74 (2006) 103521 [hep-ph/0602187] [INSPIRE].

[28] S. Baum, R. Catena, J. Conrad, K. Freese and M.B. Krauss, Determining dark matter properties with a XENONnT/LZ signal and LHC Run 3 monojet searches, Phys. Rev. D 97 (2018) 083002 [arXiv:1709.06051] [INSPIRE].

[29] J.B. Dent, L.M. Krauss, J.L. Newstead and S. Sabharwal, General analysis of direct dark matter detection: From microphysics to observational signatures, Phys. Rev. D 92 (2015) 063515 [arXiv: 1505.03117] [INSPIRE].

[30] R. Catena, J. Conrad and M.B. Krauss, Compatibility of a dark matter discovery at XENONnT or LZ with the WIMP thermal production mechanism, Phys. Rev. D 97 (2018) 103002 [arXiv: 1712.07969] [INSPIRE].

[31] W. Kilian, T. Ohl and J. Reuter, WHIZARD: Simulating Multi-Particle Processes at LHC and ILC, Eur. Phys. J. C 71 (2011) 1742 [arXiv:0708.4233] [INSPIRE].

[32] M. Moretti, T. Ohl and J. Reuter, O'Mega: An Optimizing matrix element generator, hep-ph/0102195 [INSPIRE].

[33] A. Buckley et al., LHAPDF6: parton density access in the LHC precision era, Eur. Phys. J. C 75 (2015) 132 [arXiv: 1412.7420] [INSPIRE].

[34] T. Sjöstrand, S. Mrenna and P.Z. Skands, A Brief Introduction to PYTHIA 8.1, Comput. Phys. Commun. 178 (2008) 852 [arXiv:0710.3820] [INSPIRE].

[35] DELPHES 3 collaboration, DELPHES 3, A modular framework for fast simulation of a generic collider experiment, JHEP 02 (2014) 057 [arXiv:1307.6346] [INSPIRE].

[36] M. Cacciari, G.P. Salam and G. Soyez, FastJet User Manual, Eur. Phys. J. C 72 (2012) 1896 [arXiv: 1111.6097] [INSPIRE].

[37] R. Brun and F. Rademakers, ROOT: An object oriented data analysis framework, Nucl. Instrum. Meth. A 389 (1997) 81 [INSPIRE].

[38] S. Baum, R. Catena and M.B. Krauss, Constraints on Simplified Models for Dark Matter from LHC Dijet Searches, arXiv:1812.01585 [INSPIRE]. 
[39] A.L. Fitzpatrick, W. Haxton, E. Katz, N. Lubbers and Y. Xu, The Effective Field Theory of Dark Matter Direct Detection, JCAP 02 (2013) 004 [arXiv:1203.3542] [INSPIRE].

[40] R. Catena, K. Fridell and V. Zema, Direct detection of fermionic and vector dark matter with polarised targets, JCAP 11 (2018) 018 [arXiv:1810.01515] [INSPIRE].

[41] F. Bishara, J. Brod, B. Grinstein and J. Zupan, DirectDM: a tool for dark matter direct detection, arXiv: 1708.02678 [INSPIRE].

[42] R. Catena and B. Schwabe, Form factors for dark matter capture by the Sun in effective theories, JCAP 04 (2015) 042 [arXiv: 1501.03729] [INSPIRE].

[43] R. Catena, Prospects for direct detection of dark matter in an effective theory approach, JCAP 07 (2014) 055 [arXiv: 1406. 0524] [INSPIRE].

[44] R. Catena and P. Gondolo, Global fits of the dark matter-nucleon effective interactions, JCAP 09 (2014) 045 [arXiv: 1405. 2637] [inSPIRE].

[45] M. Cirelli, E. Del Nobile and P. Panci, Tools for model-independent bounds in direct dark matter searches, JCAP 10 (2013) 019 [arXiv:1307.5955] [INSPIRE].

[46] N. Anand, A.L. Fitzpatrick and W.C. Haxton, Weakly interacting massive particle-nucleus elastic scattering response, Phys. Rev. C 89 (2014) 065501 [arXiv:1308.6288] [INSPIRE].

[47] B.A. Dobrescu and I. Mocioiu, Spin-dependent macroscopic forces from new particle exchange, JHEP 11 (2006) 005 [hep-ph/0605342] [INSPIRE].

[48] J. Fan, M. Reece and L.-T. Wang, Non-relativistic effective theory of dark matter direct detection, JCAP 11 (2010) 042 [arXiv: 1008.1591] [INSPIRE].

[49] G. Cowan, K. Cranmer, E. Gross and O. Vitells, Asymptotic formulae for likelihood-based tests of new physics, Eur. Phys. J. C 71 (2011) 1554 [Erratum ibid. C 73 (2013) 2501] [arXiv: 1007.1727] [INSPIRE].

[50] CMS collaboration, Search for narrow and broad dijet resonances in proton-proton collisions at $\sqrt{s}=13 \mathrm{TeV}$ and constraints on dark matter mediators and other new particles, HEPData Repository, based on JHEP 08 (2018) 130 [DOI:10.17182/hepdata.83912].

[51] T.D.P. Edwards, B.J. Kavanagh and C. Weniger, Assessing Near-Future Direct Dark Matter Searches with Benchmark-Free Forecasting, Phys. Rev. Lett. 121 (2018) 181101 [arXiv: 1805.04117] [INSPIRE].

[52] CMS collaboration, Search for high-mass resonances in dilepton final states in proton-proton collisions at $\sqrt{s}=13 \mathrm{TeV}$, JHEP 06 (2018) 120 [arXiv: 1803.06292] [INSPIRE]. 